\title{
An Agent-Based Decision Support System for Ecological-Medical Situation Analysis
}

\author{
Marina V. Sokolova ${ }^{1,2}$ and Antonio Fernández-Caballero ${ }^{1}$ \\ ${ }^{1}$ Universidad de Castilla-La Mancha, Escuela Politécnica Superior de Albacete \& \\ Instituto de Investigación en Informática de Albacete, 02071-Albacete, Spain \\ \{marina, caballer\}@dsi.uclm.es \\ ${ }^{2}$ Kursk State Technical University, Kursk, ul.50 Let Oktyabrya, 305040, Russia
}

\begin{abstract}
This paper presents an architecture of an agent-based decision support system (ADSS) for ecological-medical situation assessment. The system receives statistical information in form of direct and indirect pollution indicator values. The ultimate goal of the modeled multi-agent system (MAS) is to evaluate the impact of the exposure to pollutants in population health. The proposed ADSS interacts with humans in realtime "what-if" scenarios, providing the user with evidence for optimal decision making. A detailed description of all the agents and their BDI (beliefs, desires, intentions) cards is presented.
\end{abstract}

\section{Introduction}

During our lives we are open to environmental air, water, food and soil contaminants and pay progress with our health. Our research serves to the purpose of the evaluation of population exposures caused by indoor and outdoor pollution. The population exposures permit assessing the impact of environmental pollution into population morbidity in general and the contribution of each contaminant in particular by means of an agent-based decision support system (ADSS). Different research institutes and organizations confirm the ecological contribution to illness and death in adults and children [1]. The World Health Organization (WHO) declared the necessity of broader analysis of environmental risk factors over public health, with a strong emphasis on mortality [2.

To date there are some known applications of multi-agent systems (MAS) for situation assessment, including environment and public health. For example, in a recent work [3] the agent-based model for estimating residential water demand was described; the agent-based approach was used to create a system as support in clinical management [4; data mining techniques for knowledge discovery within MAS was used for early diagnostics to early intervention to developmentally-delayed children [5]. In [6] the MAS paradigm was introduced with the objective of revealing correlations between human health and environmental stress factors (traffic activity, meteorological data, and noise monitoring information) using a wide range of data mining (DM) methods, including regression analysis, neural networks, ANOVA, and others. 


\section{The ADSS Functionality and Design Characteristics}

We suggest implementing an agent-oriented software system, dedicated to ecological / medical situation estimation. The system receives retrospective statistical information in form of direct indicator values - water pollution, solar radiation and in form of indirect indicator values - types and number of vehicles used, energy used annually and energy conserved, types and quantity of used fuel, etc. [7. The indirect indicators are utilized in accordance with ISO 14031 "Environmental Performance Evaluation" standard in order to estimate air and soil pollution 8. The population exposure is registered as number of morbidity cases, with respect to International Statistical Classification of Diseases and Related Health Problems, 10th review (ICD-10) 9].

The system is aimed to fulfill the enumeration of general goals, subdivided into plans and actions, in MAS terminology, as shown in Fig. 1. This enumeration includes a set of scenarios starting from meta-ontology creation and finishing

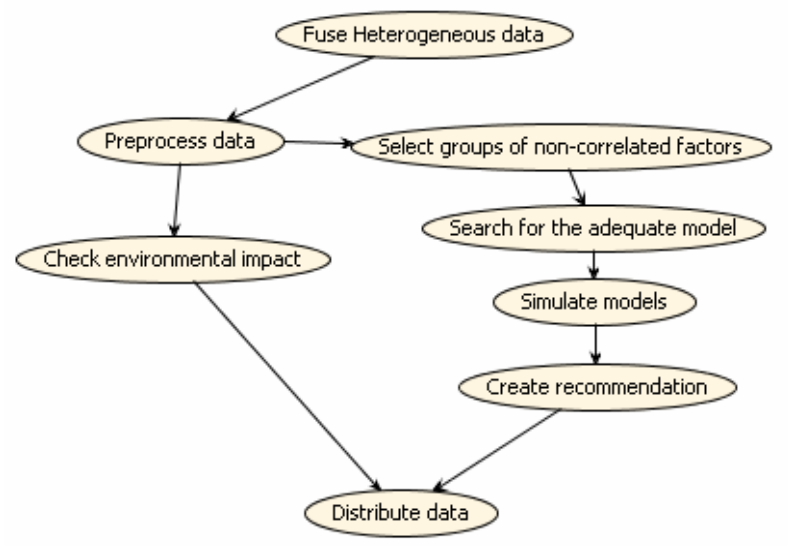

Fig. 1. The ADSS Goals Overview diagram

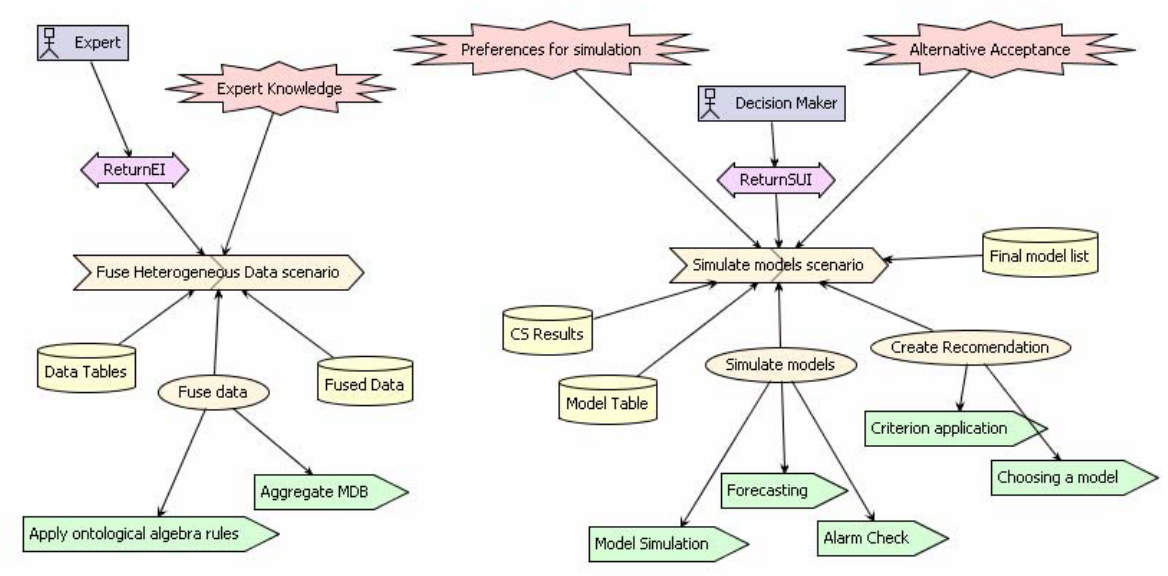

Fig. 2. The ADSS Analysis Overview diagram 


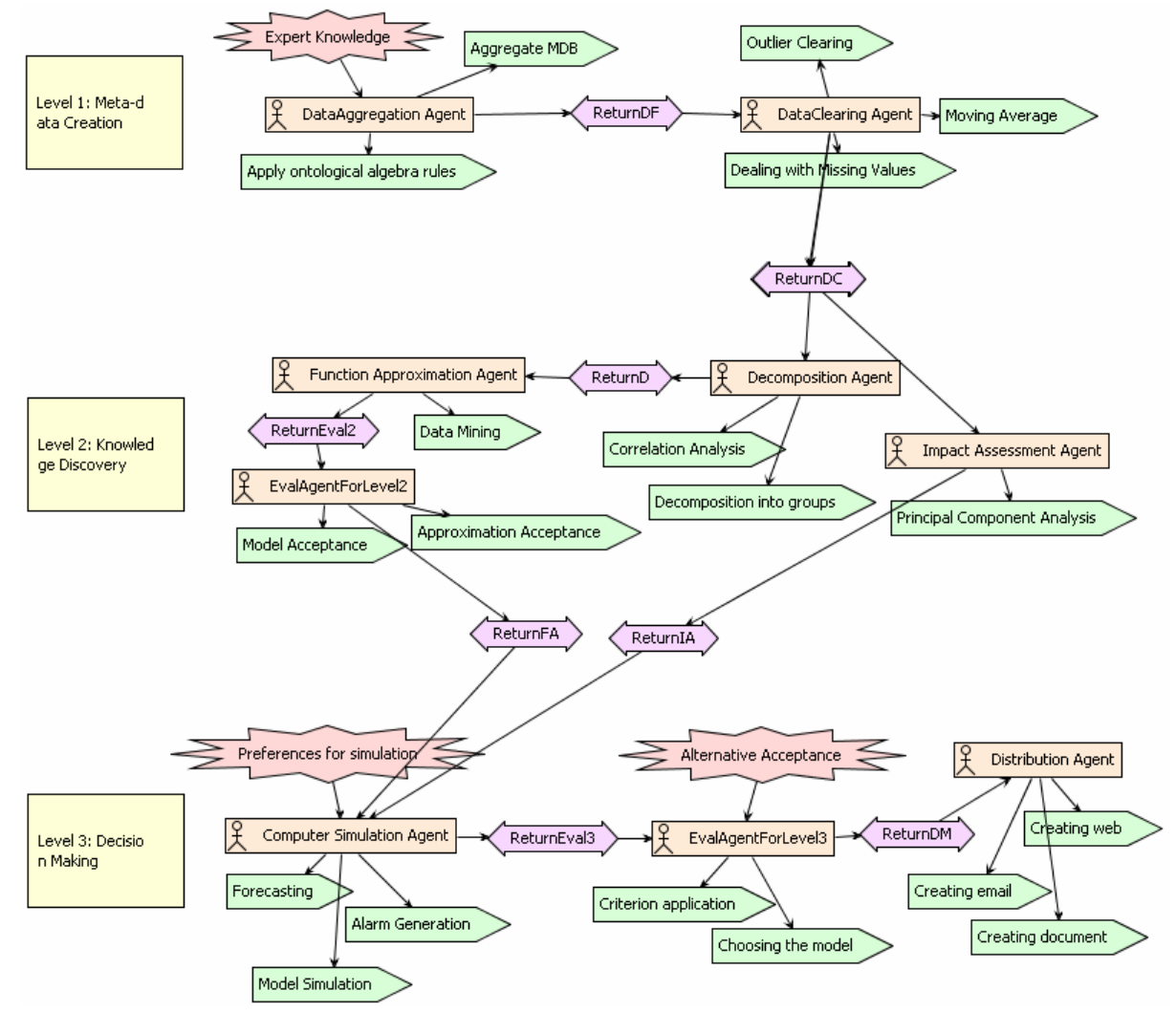

Fig. 3. The ADSS System Overview diagram

with decision making and data distribution. The ADSS design has entirely been modeled by means of Prometheus methodology 10] through Prometheus Design Tool [11. The tool provides the possibility of checking the consistency of the created system and of generating a skeleton code for JACK Intelligent Agents 12 development tool, as well as design reports in HTML.

The process of creatiing a multi-system architecture, in accordance with the Prometheus methodology, consists of three phases, which are:

1. System specification, aimed to the identification of multi-agent system entities, such as actors, system goals, scenarios, actions, percepts and roles.

2. High-level (architectural) design, which is centered in the description of agent-role coupling, general system structure and interaction protocols.

3. Detailed design, in which each agent is described in detail in terms of capabilities, events, plans and data.

The goals drawn in Fig. 1 repeat the main points of a traditional decisionmaking process, which includes the following steps: (1) problem definition, (2) 


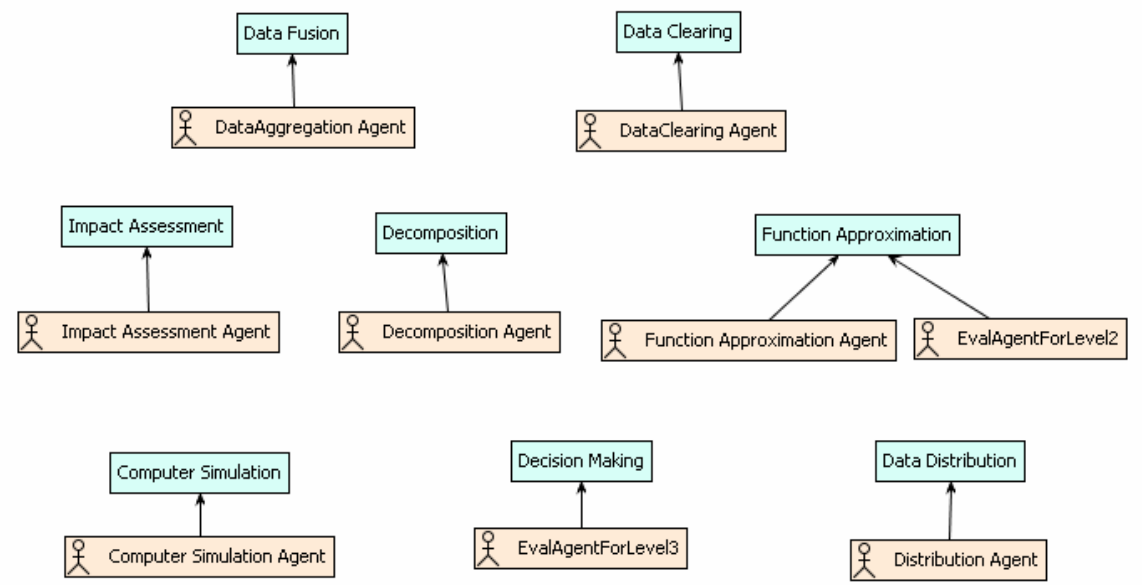

Fig. 4. The ADSS Agent-Roles Coupling diagram

information gathering, (3) alternative actions identification, (4) alternatives evaluation, (5) best alternative selection, and, (6) alternative implementation. Phases 1 and 2 are performed on the initial step, when the expert information and initial retrospective data is gathered. Phases 3 to 5 are solved by means of the ADSS, and phase 6 is supposed to be undertaken by the decision maker.

Being implemented by means of the Prometheus Design Tool, the Analysis Overview Diagram of the ADSS enables seeing the high-level view composed of external actors, key scenarios and actions (see Fig. 2). The proposed ADSS presupposes communication with two actors. One actor is named as "Expert" and it embodies the external entity which possesses the information about the problem area - in more detail, it includes the knowledge of the domain of interest represented as an ontology - and delivers it through protocol ReturnEI to the ADSS. The second actor, named "Decision Maker", is involved in an interactive process of decision making and choosing the optimal alternative. This actor communicates with agents by message passing through protocol ReturnSUI, stating the model, simulation values, prediction periods, levels of variable change, etc. It accepts the best alternative in accordance with its believes and the ADSS recommendation model.

The initial analysis of the system results in obtaining and describing the system roles and protocols. The architecture of the ADSS is offered in Fig. 3. There, the proposed system is logically and functionally divided into three layers; the first is dedicated to meta-data creation (information fusion), the second is aimed to knowledge discovery (data mining), and the third layer provides real-time generation of alternative scenarios for decision making. Fig. 3 also provides a look on connections between agents with correspondent interactions and undertaken sets of actions. This view gives a sufficient understanding of the entire system design.

The system resembles a typical organizational structure, as the agents are strictly dedicated to work with the stated sets of data sources, solve the particular tasks and are triggered when all the necessary conditions are fulfilled and 
there are positive messages from previously executed agents [13. The system includes a set of roles, correlated with the main system functions and a set of agents related to each role (see Fig. 4). Actually, mostly every agent is associated to one role; only in case of "Function Approximation" role, there are two agents, one for data mining, and the other one for validation.

\section{Description of the ADSS Agents}

Each type of agent has its own sphere of awareness (competence), belonging to some agent type. Agents possess different characteristics and use various techniques, as summarized in detail in Table 1, where agent types are given in columns, and the tasks they solve are labeled by a "+" sign. We have accepted the following abbreviations: DBA - DB Handling agents, AA - Analysis agents, EA - Evaluation agents, SA - Simulation agents, DA - Distribution agents. The types of agents are described in detail in the following section of the article. We also represent for every agent its BDI (beliefs, desires, intentions) card, which was adapted from [14].

Table 1. Task Delegation in the ADSS

\begin{tabular}{|c|c|c|c|c|c|c|c|}
\hline Level & Role & Task & $\overline{\mathrm{DBA}}$ & $\overline{\mathrm{AA}}$ & $\overline{\mathrm{EA}}$ & $\overline{\mathrm{SA}}$ & $\overline{\mathrm{DA}}$ \\
\hline \multirow[t]{5}{*}{ Level 1} & \multirow[t]{2}{*}{ Data Fusion } & DB Transformation & + & - & - & - & - \\
\hline & & Meta-data Fusion & + & - & - & - & - \\
\hline & \multirow[t]{3}{*}{ Data Clearing } & Anomalies Elimination & + & - & - & - & - \\
\hline & & Missing Values Treatment & + & - & - & - & - \\
\hline & & Smoothing & + & - & - & - & - \\
\hline \multirow[t]{6}{*}{ Level 2} & \multirow[t]{2}{*}{ Decomposition } & Correlation Analysis & - & + & - & - & - \\
\hline & & Subsets Selection & - & + & - & - & - \\
\hline & \multirow[t]{3}{*}{ Function Approximation } & Data Mining & - & + & - & - & - \\
\hline & & Model Evaluation & - & - & + & - & - \\
\hline & & Approximation Acceptance & - & - & + & - & - \\
\hline & Impact Assessment & Principal Component Analysis & - & + & - & - & - \\
\hline \multirow[t]{8}{*}{ Level 3} & \multirow{3}{*}{ Computer Simulation } & Simulation & - & - & - & + & - \\
\hline & & Forecasting & - & - & - & + & - \\
\hline & & Alarm Check & - & - & - & + & - \\
\hline & \multirow[t]{2}{*}{ Decision Making } & Criterion Calculation & - & - & + & - & - \\
\hline & & Model Selection & - & - & + & - & - \\
\hline & \multirow[t]{3}{*}{ Distribution } & Creation of Documents & - & - & - & - & + \\
\hline & & Creation of Web Source & - & - & - & - & + \\
\hline & & Creation of E-mails & - & - & - & - & + \\
\hline
\end{tabular}

\subsection{DB Handling Agent Type}

The DB Handling agent type contains two agents, the Data Aggregation agent and the Data Clearing agent. The difference between their competence areas has been introduced in their names; the first one is oriented to data fusion, and the second one is focused on data preprocessing. 
Table 2. BDI card for the Data Aggregation agent

\begin{tabular}{|l|l|}
\hline Desires & Create \\
\hline Pre-condition & Expert information is received \\
\hline Beliefs & Problem ontology \\
\hline Post-condition & Termination message \\
\hline Collaborator & - \\
\hline Intentions & Search data in data sources according to ontology \\
\cline { 2 - 2 } & Fuse data into meta-schema \\
\cline { 2 - 2 } & Send a termination message to the Data Clearing agent \\
\hline Type & DB Handling agent \\
\hline
\end{tabular}

Table 3. BDI card for the Data Clearing agent

\begin{tabular}{|l|l|}
\hline Desires & Replace data \\
\hline Pre-condition & Message from Data Aggregation agent \\
\hline Beliefs & Meta-DB \\
\hline Post-condition & Termination message \\
\hline Collaborator & - \\
\hline \multirow{5}{*}{ Intentions } & Eliminate outliers and anomalies \\
\cline { 2 - 2 } & Treat missing values \\
\cline { 2 - 2 } & Smooth time series \\
\cline { 2 - 2 } & Send termination message \\
\hline Type & DB Handling agent \\
\hline
\end{tabular}

The Data Aggregation agent. The Data Aggregation agent (DAA) is responsible for initial information reception and meta-data creation. The agent plays an essential role as it has to realize the plan of important tasks, including: (1) reception of expert information and initial data, (2) scanning of data sources for selecting the values related to identical hierarchical and semantical levels of the meta-ontology, (3) pooling homogeneous data into a meta-data structure. The DAA communicates with the Data Clearing agent through communication protocol ReturnDF, sending a message that confirms meta-data creation and triggers data preprocessing.

The Data Clearing agent. The Data Clearing agent (DCA) performs all data preprocessing procedures, including outliers and anomalies detection, dealing with missing values, smoothing, normalization, etc. The final meta-data consists of sequences of ordered indicator values measured at equal time intervals (timeseries).

\subsection{Analysis Agent Type}

The Impact Assessment agent. The single agent that belongs to this type, Impact Assessment agent (IAA), solves the principal component analysis (PCA) procedure for every type of disease and the totality of pollutant indicators. The outcomes are interpreted as the determination of the most influencing subsets of pollutants for every nosology. It is message-triggered from the DCA and after being executed it creates a file with the results of its work. The IAA delivers messages to Simulation agent through protocol ReturnIA.

The Decomposition agent. The main task of the Decomposition agent (DA) is to separate the totality of factors (pollution indicators) and output variables 
Table 4. BDI card for the Impact Assessment agent

\begin{tabular}{|l|l|}
\hline Desires & Execute \\
\hline Pre-condition & Message from Data Clearing agent \\
\hline Beliefs & Meta-DB \\
\hline Post-condition & Termination message \\
\hline Collaborator & - \\
\hline Intentions & Execute PCA procedure \\
\hline Type & Analysis agent \\
\hline
\end{tabular}

Table 5. BDI card for the Decomposition agent

\begin{tabular}{|l|l|}
\hline Desires & Execute \\
\hline Pre-condition & Message from Data Clearing agent \\
\hline Beliefs & Meta-DB \\
\hline Post-condition & Termination message \\
\hline Collaborator & - \\
\hline Intentions & Executes procedures of correlation analysis \\
\cline { 2 - 2 } & Select subsets for every output variable \\
\cline { 2 - 2 } & Send termination message \\
\hline Type & Analysis agent \\
\hline
\end{tabular}

(morbidity) into subsets (commonly intersected) with respect to every output variable (nosology). Decomposition is aimed to select for every output variable those factors that (1) do not correlate significantly with the output variable in order to avoid inter-correlation, or, (2) do not correlate significantly between themselves - in order to avoid multi-colinearity. The DA creates a correlation matrix and analysis it. The Decomposition agent creates an output source used then by Simulation agent and Distribution agent.

The Function Approximation agent. The Function Approximation agent (FAA) carries out a core ability: it explores unknown relationships, trends and connections in data, received from the DA agent. The FAA is competent in applying data mining (DM) techniques, including theory-driven methods (linear, non linear regression, ANOVA, etc.) as well as data-driven methods (neural networks, decision trees, rules, etc.). Taking into account the nature of metadata base, we state the following requirements to DM algorithms: robustness, ability to work with missing data values, and ability to deal with short data sets (as data are usually being registered once a year; so, in better case we can operate with data sets including up to 50 values). As DM techniques satisfy these requirements, they will be used.

\subsection{Evaluation Agent Type}

As shown before in Table 1, the Evaluation agent type is involved in two roles: "Function Approximation" and "Decision Making", which also belong to different levels. In role "Function Approximation" the Evaluation agent is thought to calculate statistical performance characteristics for the model, created by the FAA, and the model's approximation abilities. To do it, we approximate initial data by the models, compare the real and the approximated data sets and evaluate the 
Table 6. BDI card for the Function Approximation agent

\begin{tabular}{|l|l|}
\hline Desires & Execute \\
\hline Pre-condition & Message from Decomposition agent \\
\hline Beliefs & Meta-DB, Grouping Table \\
\hline Post-condition & Termination message \\
\hline Collaborator & - \\
\hline Intentions & Apply Data Mining technique \\
\cline { 2 - 2 } & Send a message to Evaluation agent \\
\hline Type & Analysis agent \\
\hline
\end{tabular}

Table 7. BDI card for the Evaluation agent for level 2

\begin{tabular}{|l|l|}
\hline Desires & Execute \\
\hline Pre-condition & Message from the Function Approximation agent \\
\hline Beliefs & Meta-DB, Models table \\
\hline Post-condition & Termination message \\
\hline Collaborator & - \\
\hline Intentions & Model evaluation \\
\cline { 2 - 2 } & Model approximation acceptance \\
\cline { 2 - 2 } & Send a termination message \\
\hline Type & Evaluation agent \\
\hline
\end{tabular}

Table 8. BDI card for the Evaluation agent for level 3

\begin{tabular}{|l|l|}
\hline Desires & Execute \\
\hline Pre-condition & Message from the Function Approximation agent \\
\hline Beliefs & CS results table, Final model list \\
\hline Post-condition & Termination message \\
\hline Collaborator & Computer Simulation agent \\
\hline \multirow{3}{*}{ Intentions } & Game theory criteria application \\
\cline { 2 - 2 } & Best model selection \\
\cline { 2 - 2 } & Send a termination message \\
\hline Type & Evaluation agent \\
\hline
\end{tabular}

performance statistics. In role "Decision Making" the Evaluation agent applies decision making theory criteria (Bayes, minimax, Hurwicz, and so on).

\subsection{Simulation Agent Type}

The Computer Simulation agent. Actually, there is one agent that belongs to this type, namely the Computer Simulation agent (CSA), which works with models created by the FAA and accepted by an Evaluation agent. The simulation process involves the interaction with the user. During this process the CSA learns the user's preferences: the model to be simulated, the factors the user is interested in analyzing, the forecast period, etc. The CSA simulates a model within capability "Simulation", compares the outputs with the permitted and hazardous levels within capability "Alarm Check" and shows the results to the user in order to be revised and accepted, or refined and repeated. This creates a decision support system, which helps the user in decision making by providing several alternatives. 
Table 9. BDI card for the Computer Simulation agent

\begin{tabular}{|l|l|}
\hline Desires & Execute \\
\hline Pre-condition & $\begin{array}{l}\text { Message from the Evaluation agent for level } 2 \\
\text { and from Impact Assessment agent }\end{array}$ \\
\hline Beliefs & Models table, Alarm levels \\
\hline Post-condition & Termination message \\
\hline Collaborator & - \\
\hline Intentions & Simulation \\
\cline { 2 - 2 } & Checking the permitted levels \\
\cline { 2 - 2 } & Send a termination message \\
\hline Type & Simulation agent \\
\hline
\end{tabular}

\subsection{Distribution Agent Type}

The Data Distribution agent (DDA) emphasizes in data visualization and delivering to the user. The revealed information is transformed by the DDA into multiple understandable forms. These tasks are performed by the Data Distribution agent, which operates by combining textual and graphical descriptions of recommendations. This information jointly with computer simulation results for chosen models (including forecast and alarm check) are delivered. This includes impact assessment outcomes (which discover the exposure of urban pollution on the morbidity of people), correlation results (which reveal dependencies between pollutants and different types of diseases), and, optionally, those models recommended by the system to be accepted as optimal for regional development and situation correction.

Table 10. BDI card for the Data Distribution agent

\begin{tabular}{|l|l|}
\hline Desires & Create \\
\hline Pre-condition & Message from Evaluation agent for level 3 \\
\hline Beliefs & Final model list, Correlation table, PCA results \\
\hline Post-condition & Termination message \\
\hline Collaborator & - \\
\hline Intentions & $\begin{array}{l}\text { Create textual document } \\
\text { Create web-source } \\
\text { Create and send e-mail }\end{array}$ \\
\hline Type & Distribution agent \\
\hline
\end{tabular}

\section{Conclusions}

The agent-based decision support system (ADSS) introduced in this paper provides all the necessary steps for standard managerial decision procedure by utilizing intelligent agents. The levels of the system architecture, logically and functionally connected, have been presented. Real-time interaction with the user provides a range of possibilities in choosing one course of action from among several alternatives, which are generated by a system through directed data mining and computer simulation. The system is aimed to regular usage for adequate and effective management by responsible municipal and state government authorities.

The proposed ADSS architecture may be generalized and applied in closely related areas, such as economics, sociology, public health, etc. Some specific DM 
techniques, for example, econometric system dynamic models, can be used in these kinds of applications.

\section{Acknowledgements}

Marina V. Sokolova is the recipient of a Postdoctoral Scholarship (Becas MAE) awarded by the Agencia Española de Cooperación Internacional of the Spanish Ministerio de Asuntos Exteriores y de Cooperación.

\section{References}

1. Kaiser R., Romieu I., Medina S., Schwartz J., Krzyzanowski M., Künzli N. (2004) Air pollution attributable postneonatal infant mortality in U.S. metropolitan areas: A risk assessment study. Environmental Health: A Global Access Science Source, vol.3, no. 1 , p. 4.

2. World Health Organization. http://www.who.int/en/

3. Athanasiadis, I.N., Mentes, A.K., Mitkas, P.A., Mylopoulos, Y.A. (2005). A hybrid agent-based model for estimating residential water demand. Simulation, vol. 81, no. 3, pp. 175-187.

4. Foster D., McGregor C., Samir E.S. (2006) A survey of agent-based intelligent decision support systems to support clinical management and research. http://www.diee.unica.it/biomed05/pdf/W22-102.pdf.

5. Chang, C.L. (2006). A study of applying data mining to early intervention for developmentally-delayed children. Expert Systems with Applications. In press.

6. Chen, H., Bell, M. (2002). Instrumented city database analysts using multi-agents. Transportation Research, Part C, vol. 10, pp. 419-432.

7. Sokolova, M.V., Fernández-Caballero, A. (2007). A multi-agent architecture for environmental impact assessment: Information fusion, data mining and decision making. 9th International Conference on Enterprise Information Systems, ICEIS 2007.

8. ISO 14031:1999. Environmental management - Environmental performance Guidelines. http://www.iso.org/

9. International Classification of Diseases (ICD). http://www.who.int/classifications/icd/en/

10. Padgham, L., Winikoff, M. (2004). Developing Intelligent Agent Systems: A Practical Guide. John Wiley and Sons.

11. Prometheus Design Tool (PDT). http://www.cs.rmit.edu.au/agents/pdt/

12. Jack Intelligent Agents. http://www.agent-software.com/shared/home/

13. Weiss, G. (2000). Multiagent Systems: A Modern Approach to Distributed Artificial Intelligence. The MIT Press.

14. Jo, C.H., Einhorn, J.M. (2005) A BDI agent-based software process. Journal of Object Technology, vol. 4, no. 9, pp. 101-121. 